\title{
THE SOIL MICROBIAL COENOSIS STATE UNDER VARIOUS CROPS IN CASE OF INTRODUCING THE MIXTURE OF SILICON-CONTAINING MINERALS
}

\author{
N. E. Ellanska, N. V. Zaimenko, O. P. Yunosheva
}

Among the important factors that restrict area use in open ground and areas in closed ground, adverse reaction of soil solution (first of all the existence of acidic environment that is decreased concentrations of $\mathrm{H}^{+}$and $\mathrm{Al}^{3+}$ ) should be noted. According to the State Agency of Land Resources of Ukraine the area of acid soils is about 9.5 million ha. In recent years in Ukraine, as well as worldwide, secondary soil acidification processes caused by acid rains and unbalanced use of fertilizers have expanded. Consequently, even neutral in nature black soils are being acidified. The reduction of soil acidity leads to changes in its physical and chemical features, violation of functional and structural properties of biota and, eventually, affects the productivity and vitality of plants [1].

The issue attracts the attention of many scientists who are engaged in finding the ways for fertility rebirth, including the means for acid soils amelioration (local, phytobiological) [2].

One of the environmentally friendly ways for increasing agricultural crops productivity on such soils is the use of natural silicon-containing minerals of fine fraction with high sorption capacity (analcime, tripoli, etc.) [3]. It is known that silicon is marked by positive impact on functional status of living organisms, helps stimulate root development, improves soil agrophysical performance by increasing the moisture capacity, creates a chemical depot of macro- and microelements, increases the resistance of plants to abiotic stress factors $[4 ; 5]$. Biological effect of agents is enhanced by combined use of low doses of silicon-containing minerals nanoparticles and organic substances.

Soil microorganisms perform different environmental functions, the major of which provide certain stages of circulation of nutrients and maintain soil ecosystem homeostasis. The effect of silicon-containing compounds on soil microbiological activity is of great interest, because even a small change in the environment, common for microorganisms, affects the intensity of many microbiological processes, due to which the effect of microbiota on metabolism in plants changes $[4 ; 6]$. Therefore, the study of such dynamic system as microbial communities, the impact of various factors on them, has great significance [7].

In view of the above, the objective of our research work was to study soil microbiological condition under various crops in case of silicon-containing compounds introducing.

Materials and methods. Soil samples, selected on research fields of the Institute of bioenergy crops and sugar beets of NAAS (v. Solonivka of Kaharlyk district of Kyiv region) located on low-humus black soil ( $\mathrm{pH}$ 5.48-6.22; humus content $2.0-2.1 \%$ ) served as research material. In field experiments was studied the effect of four mixtures based on natural silicon-containing minerals (analcime - mineral from the group of water zeolites of silicate class, chemical formula $\mathrm{Na}\left[\mathrm{AlSi}_{2} \mathrm{O}_{6}\right] \cdot \mathrm{H}_{2} \mathrm{O}$ and tripoli fine-pored opal sediment of diatomite class) produced in Ukraine, sapropel and peat (300 $\mathrm{kg} / \mathrm{ha}$ ) on the development of soil microbiota in the conditions of soil acidification (Table 1). Samples were taken in two terms - in 2 (I) and 4 (II) months after the mixtures were applied. Sugar beet (Standart and Kvarta hybrids), corn (Orbit hybrid), soybeans (Suziria hybrid) were cultivated on research areas.

Table 1. The content of silicon-containing

minerals mixture with organic additives

\begin{tabular}{|l|l|}
\hline No. & \multicolumn{1}{|c|}{ Content } \\
\hline 1 & control \\
\hline 2 & $70 \%$ tripoli, 30\% high-bog peat \\
\hline 3 & $70 \%$ tripoli, 30\% analcime \\
\hline 4 & $50 \%$ tripoli, $15 \%$ analcime, \\
& $25 \%$ potassium silicate, $10 \%$ sapropel \\
\hline 5 & $7 \%$ tripoli, 3\% analcime, $90 \%$ sapropel \\
\hline
\end{tabular}

Microbiological analyzes were carried out by conventional methods [8]. On meat-and-peptone agar (MPA) the number of amonificators was taken into account, on starch-ammonium agar (SAA) - microorganisms that absorb mainly mineral nitrogen and 
actinomycetes, the number of micromycetes - on Czapek's medium. The number of nitrogen-fixing microorganisms was determined by the method of applications on the surface agar Ashby medium [9]. The ratio of specific eco-trophic groups of microorganisms (coefficient of mineralization-immobilization) was calculated by K. I. Andreiuk et al. [10], the rate of organic matter transformation was determined by V. D. Mukha [11].

Statistical data processing was performed using the software package Microsoft Excel 2007.

Thus, we can conclude that quantitative analysis of different eco-trophic groups of microorganisms and their activity allows to estimate the direction of humus-creating processes that occur in soil in case of silicon-containing compounds introducing. The predominance of any mixture formula over others was not marked. The specificity of action of mixtures on rhisospheric microbiota of studied crops was detected. Positive effect on soil microbiota of sugar beet was made by mixtures No. 2 and No. 4. Rhizospheric soil microorganisms of soybeans and corn reacted most sensitively, especially in case of introducing of mineralized sapropels. The processes of soil organic matter mineralization were quite balanced. The difference in the number of microorganisms under different hybrids of sugar beet was detected. Probably, a significant difference in root secretions of genetically diverse hybrids ("Standart" - German selection, and "Kvarta" - domestic) determines the specificity of microbocenoces formation.

Introducing of silicon-containing compounds first leads to "imbalance" in microbial coenosis, and eventually stabilizes and activates microorganisms. Harmless of minerals for soil microbiotawas proved, as evidenced by high level of total number and absence of sharp fluctuations of studied groups of microorganisms. Studies contribute to the importance of silicon-containing compounds in enhancing the adaptive capacity of plants to soil acidification; they likely contribute to optimization of conditions for nutrients receipt and humification processes flow. It was found that in the presence of silicon-containing compounds (especially No. 1 and No. 2) the increase in productivity of all studied crops was observed: sugar beet by $9.7-41 \%$, soybeans - by $6.2-50 \%$, corn $-5.5-30.6 \%$. 